\title{
Prognosis Implication of a Novel Metabolism-Related Gene Signature in Ewing Sarcoma
}

\author{
Zhuo-Yuan Chen $\mathbb{D}^{1},{ }^{1,2}$ Huiqin Yang, ${ }^{1,2}$ Jie Bu $\mathbb{D}^{\circ},{ }^{3}$ Qiong Chen, ${ }^{4}$ Zhen Yang, ${ }^{5}$ and Hui Li ${ }^{1,2}$ \\ ${ }^{1}$ Department of Orthopedics, The Second Xiangya Hospital, Central South University, 139th Renmin Middle Road, Changsha, \\ Hunan, China \\ ${ }^{2}$ Orthopedic Biomedical Materials Engineering Laboratory of Hunan Province, 139th Renmin Middle Road, Changsha, \\ Hunan, China \\ ${ }^{3}$ Hunan Cancer Hospital and the Affiliated Cancer Hospital of Xiangya School of Medicine, Central South University, \\ 283 Tongzipo Road, Changsha, Hunan, China \\ ${ }^{4}$ Department of Ultrasound, Wuhan No. 1 Hospital, 215 Zhongshan Dadao, Wuhan, Hubei, China \\ ${ }^{5}$ Department of Radiology, Hubei Cancer Hospital, 116 Zhuodaoquan South Road, Wuhan, Hubei, China
}

Correspondence should be addressed to Hui Li; lihuix@csu.edu.cn

Received 27 August 2021; Accepted 19 November 2021; Published 10 December 2021

Academic Editor: Shinji Miwa

Copyright () 2021 Zhuo-Yuan Chen et al. This is an open access article distributed under the Creative Commons Attribution License, which permits unrestricted use, distribution, and reproduction in any medium, provided the original work is properly cited.

\begin{abstract}
Ewing sarcoma (ES) is one of the most common bone cancers in adolescents and children. Growing evidence supports the view that metabolism pathways play critical roles in numerous cancers (He et al. (2020)). However, the correlation between metabolism-associated genes (MTGs) and Ewing sarcoma has not been investigated systematically. Here, based on the univariate Cox regression analysis, we get survival genes from differentially expressed genes (DEGs) from Gene Expression Omnibus (GEO) cohort. Multivariate Cox regression analysis and least absolute shrinkage and selection operator (LASSO) regression analysis were employed to establish the MTG signature. Comprehensive survival analyses including receiver operating characteristic (ROC) curves and Kaplan-Meier analysis were applied to estimate the independent prognostic value of the signature. The ICGC cohort served as the validation cohort. A nomogram was constructed based on the risk score of the MTG signature and other independent clinical variables. The CIBERSORT algorithm was applied to estimate immune infiltration. In addition, we explored the correlation between MTG signature and immune checkpoints. Collectively, this work presents a novel MTG signature for prognostic prediction of Ewing sarcoma. It also suggests six genes that are potential prognostic indicators and therapeutic targets for ES.
\end{abstract}

\section{Introduction}

Ewing sarcoma is a primary malignancy of the bone or soft tissue [1]. It is ranked the second most prevalent bone cancer in adolescents and children [2]. Current evidence shows that metastasis of ES is still the main indicator to predict the outcomes of ES patients for lacking effective biomarkers [3]. Koustas et al. found that the 5-year survival rate of patients with metastasis is only $20 \%-45 \%$ [4]. Therefore, effective biomarkers that can accurately predict disease outcomes and offer novel therapeutic targets for Ewing sarcoma are urgently needed.
The concept of metabolic reprogramming was first put forward by Otto Warburg in 1924 [5]. Several lines of evidence have demonstrated metabolism as among the most compelling traits in cancers, for it is associated with various biological processes, including growth, proliferation, migration, and invasion, and angiogenesis $[6,7]$. Cancer cells can adjust their metabolic patterns to guarantee sufficient energy and substance. A previous study revealed that the restoration or blockage of metabolic pathways may be a promising therapeutic strategy for tumors [8]. In the context of Ewing sarcoma, a number of studies explored the importance of metabolic reprogramming in disease 
progression and prognosis. Tanner et al. [9] found that EWS/ FLI could induce diversion of metabolites toward oncogenesis-related biosynthetic pathways to meet the demand of ES cells, for example, by shunting glycolytic intermediates into serine and glycine synthesis. The inhibitors of the glycolytic enzyme lactate dehydrogenase (LDH), which are critical in cellular metabolism, are potential therapeutic targets for ES treatment [10]. Hence, uncovering metabolism-related biomarkers greatly sheds light on ES diagnosis and prognosis.

In this study, we compared the normal and ES tissues to reveal the DEGs of MTGs. Based on DEGs and prognosisrelated genes, a metabolic gene signature was established, which allowed us to stratify patients into high- and low-risk subtypes and ensure accurate prediction of survival prognosis. Furthermore, the model was validated with an independent cohort from the International Cancer Genome Consortium (ICGC) database. Analysis of the correlation between the subtypes with immune infiltration was achieved through single-sample gene set enrichment analysis (ssGSEA) to explore the roles of 24 immune cells in the metabolism-related signature. In summary, we have constructed a prognostic model that can accurately predict ES prognosis.

\section{Materials and Methods}

2.1. Data Collection. We downloaded 944 MTGs from the Kyoto Encyclopedia of Genes and Genomes (KEGG) pathways as previously described by Chao-Yang et al. [11]. The transcriptome profiles and related clinical data of ES patients were extracted from the GEO database. In total, 18 normal samples (skeletal muscle) and 64 Ewing sarcoma samples from the dataset (GSE17679) were analyzed. Fiftyfive samples contained both transcriptome and clinical data in the validation ICGC cohort.

\subsection{Construction and Validation of the Metabolism-Related} Gene Signature. The "limma" R package was employed to obtain DEGs (FDR <0.05) from the GEO dataset while genes related to overall survival (OS) were identified with univariate Cox regression analysis. By taking the intersection of DEGs and prognosis-related genes, 255 genes were identified for further analysis. According to multivariate Cox regression analysis, 6 genes were retrieved. Next, the least absolute shrinkage and selection operator (LASSO) regression analysis was employed to construct the prognostic model of the multivariate Cox regression results. The risk score was calculated using the following formula: risk score$=\sum$ icoefficient $($ genei $) \times$ expression (genei). ES patients were classified into high-risk and low-risk groups based on the median risk score. Furthermore, "survival," "survivalROC," and "stats" packages were applied to draw the Kaplan-Meier survival curves and conduct time-dependent ROC curve analysis and principal component analysis (PCA), respectively. Multivariate and univariate Cox regression analyses were applied to examine the effectiveness of the risk score as an independent prognostic indicator using the risk score and available clinicopathological data. Based on the risk score, ES patients from the ICGC dataset were classified into high- and low-risk groups and analyses were conducted to validate the effectiveness.

2.3. Gene Set Enrichment Analysis. Based on the packages "GSVA" and "GSEABase," the Gene Set Enrichment Analysis (GSEA) was applied to explore the biological functions and pathways according to the low- and high-risk groups ( $p$ value was set as 0.05 ).

2.4. Evaluation of the Prognostic Signature and the Construction of Nomogram. The predictive ability of the prognostic model for different clinicopathological characteristics was explored in the GEO cohort. Based on the "rms" R package, the nomogram was performed by the overall survival data of the GEO dataset. Moreover, the 3- and 5year calibration plots were applied to assess the accuracy of the nomogram.

2.5. Evaluation of Immune Cell Infiltration and Immune Checkpoints. We analyzed the correlation between the model and immune cell infiltration using the CIBERSORT algorithm [12] to identify the 22 immune cells' fractions in the GEO dataset. The relationship between 22 immune cells was evaluated with the Pearson correlation analysis. Furthermore, we obtained the differential immune cells by comparing the high- and low-risk groups. Later, Kaplan-Meier analysis was employed to analyze the correlation between the differential immune cells and ES patient prognosis. In addition, we estimated the association between MTG signature and immune checkpoints via the expression levels of immune checkpoint genes.

2.6. Statistical Analysis. Student's $t$-test was applied to identify the DEGs in the GEO cohort. Wilcoxon test and chisquare test were applied to analyze continuous and categorical variables, respectively. K-M curve and the log-rank test were performed to evaluate the differences in OS. All statistical analyses were performed with the $\mathrm{R}$ software (version 4.0.1), and $p<0.05$ denoted statistical significance.

\section{Results}

3.1. Identification of Metabolism-Related Prognostic DEGs and Construction of the Signature. A schematic representation of the study is illustrated in Figure 1. The characteristics of ES patients in the two datasets are listed in Table 1. The retrieved MTGs are displayed in Supplementary Table 1.

Of note, 727 MTGs were differentially expressed between the ES tissues and the normal tissues. Based on the univariate Cox regression analysis results, we obtained 297 prognostic MTGs which were related to OS. Additionally, 255 intersection results (Figure 2(a)) of DEGs and prognostic genes were illustrated in a Venn diagram. Based on the multivariate Cox regression analysis results, 255 genes were screened, 6 of which were highly related to OS. 


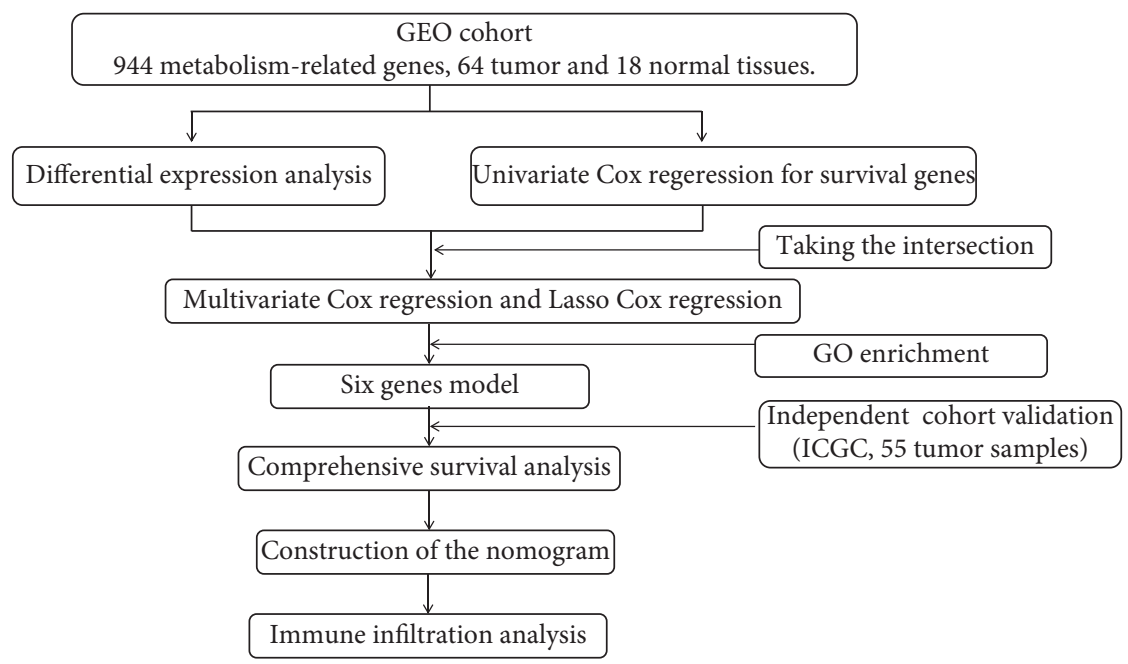

Figure 1: Flowchart of data collection and analysis.

TABle 1: The characteristics of ES patients in the two datasets.

\begin{tabular}{lcc}
\hline Variables & GEO cohort & ICGC cohort \\
\hline Numbers & 64 & 55 \\
\hline $\begin{array}{l}\text { Age, years (\%) } \\
\quad>16\end{array}$ & $36(56.25 \%)$ & $26(47.27 \%)$ \\
$\quad \leq 16$ & $28(43.75 \%)$ & $29(52.73 \%)$ \\
\hline Gender (\%) & & \\
$\quad$ Female & $20(31.25 \%)$ & $24(43.64 \%)$ \\
$\quad$ Male & $44(68.25 \%)$ & $31(56.36 \%)$ \\
\hline Survival status (\%) & & \\
$\quad$ Alive & $24(37.5 \%)$ & $27(49.09 \%)$ \\
$\quad$ Dead & $40(62.5 \%)$ & $28(50.91 \%)$ \\
\hline
\end{tabular}

Through the LASSO regression analysis (Figures 2(b) and 2(c)), we constructed the MTG prognostic signature and applied it to calculate the risk score using the following formula: risk score $=\left(0.7457^{*}\right.$ expression of PC $)+\left(-0.7713^{*}\right.$ expression of DGKA $)+\left(-0.0036^{*}\right.$ expression of $\mathrm{CPT} 1 \mathrm{~A})+\left(0.2923^{*}\right.$ expression of CHPT1 $)+\left(-0.6910^{*}\right.$ expression of NUDT12)+(0.8069* expression of PYGB). Subsequently, ES patients were classified into the low-risk group $(n=32)$ and the high-risk group $(n=32)$ according to the median cutoff risk score. As depicted in the box plot (Figure 2(d)), all the six genes were differentially expressed between the ES tissues and the normal tissues. GSEA outcomes (Figure 2(e)) indicated that the top 5 were glycine, serine, and threonine metabolism, oocyte meiosis, maturityonset diabetes of the young, cardiac muscle contraction, and vasopressin-regulated water reabsorption.

3.2. Verification of the MTG Signature. Thirty-two high-risk patients and 25 low-risk patients were found in the GEO dataset, while 30 high-risk patients and 25 low-risk patients were found in the ICGC dataset (Figures 3(a) and 3(b)). The outcomes of PCA verified that the two groups were mainly distributed in two different directions (Figures 3(c) and $3(\mathrm{~d}))$. The status of ES patients in the GEO and ICGC datasets is described in Figures 3(e) and 3(f). These data demonstrate that the high-risk groups were correlated with more deaths. Besides, Kaplan-Meier curve analysis (Figures 3(g) and 3(h)) was applied to demonstrate the OS difference between the two risk groups. The $P$ value of GEO and ICGC datasets was statistically significant $(<0.001$ and $0.020)$.

3.3. Strong Prognostic Power of the MTG Signature. Univariate and multivariate Cox regression analyses were applied to reveal the independent prognostic indicator value of risk score for ES. The univariate Cox regression analysis results (Figures 4(a) and 4(b)) showed that the risk score was significantly related to OS in the GEO dataset $(P<0.001$, $\mathrm{HR}=3.6835$, and $95 \% \mathrm{CI}=2.4016-5.6496)$ and $\mathrm{ICGC}$ dataset $\quad(p=0.016, \quad \mathrm{HR}=1.0538, \quad$ and $95 \%$ $\mathrm{CI}=1.0098-1.0997)$. The multivariate Cox regression analysis (Figures 4(c) and 4(d)) results showed that the risk score was an independent indicator in the GEO dataset $(P<0.001$, $\mathrm{HR}=4.2485$, and $95 \% \mathrm{CI}=2.5937-6.9591)$ and ICGC dataset $\quad(P=0.035, \quad \mathrm{HR}=1.0480, \quad$ and $\quad 95 \%$ $\mathrm{CI}=1.0032-1.0947)$.

Moreover, the accuracy of the signature was assessed through ROC analysis. The outcomes (Figures 4(e) and 4(f)) showed that 1-, 2-, and 3-year AUC values for the GEO cohort were $0.0856,0.810$, and 0.834 , while those for ICGC cohort was $0.0833,0750$, and 0.718 , respectively. The risk score values and the clinical features for OS were compared (Figures 4(g) and 4(h)), and the results indicated that the risk score was the best predictor.

All the outcomes demonstrated that the MTG signature was of good prognostic prediction power for ES overall survival.

3.4. The Efficiency of the MTG Signature in the GEO Cohort. To explore the efficiency of the signature, ES patients with different clinical features were divided into different groups based on age, gender, and disease state (primary tumor, metastasis, and recurrence). All the outcomes (Figure 5) 


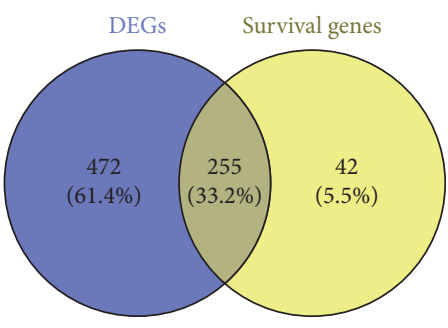

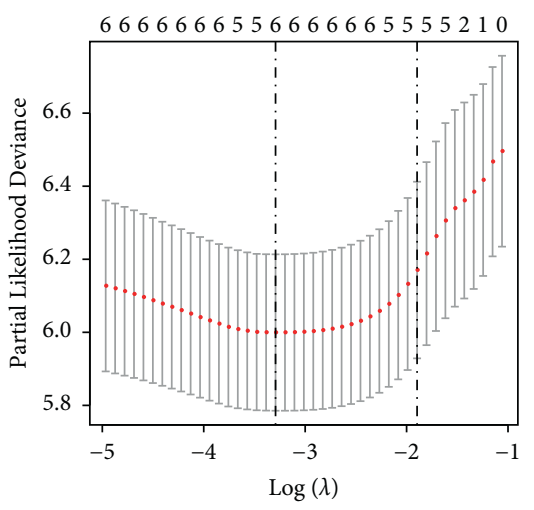

(b)

(a)
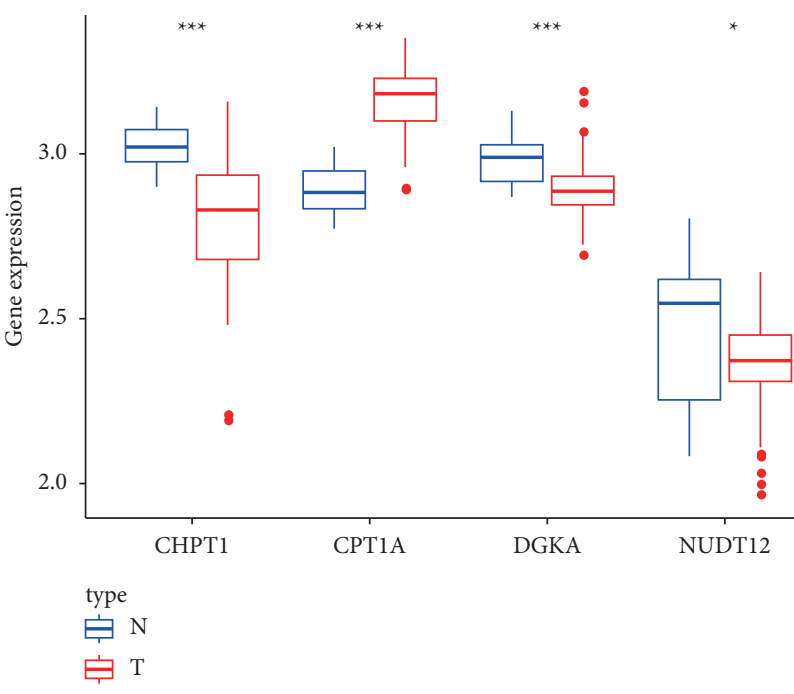

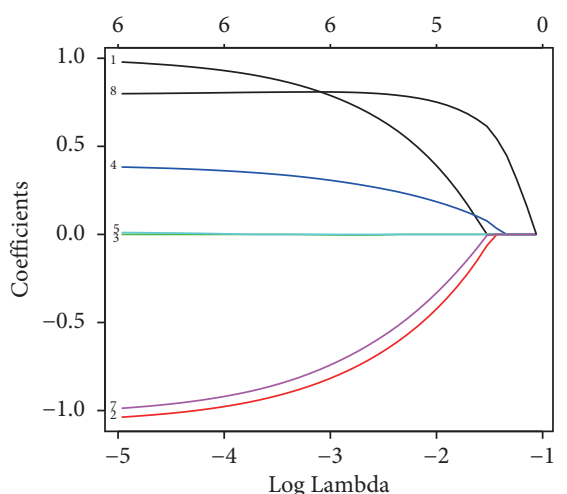

(c)

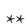

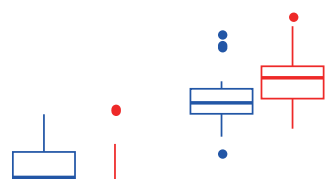

(d)

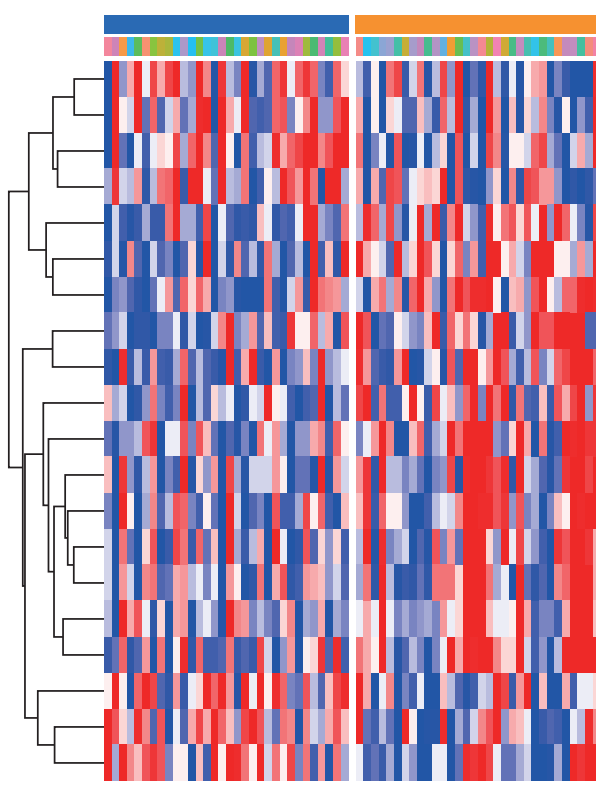

Risk

Samples

KEGG_PROPANOATE_METABOLISM

KEGG_VALINE_LEUCINE_AND_ISOLEUCINE_DEGRADATION

KEGG_RIBOFLAVIN_METABOLISM

KEGG_GLYCOSYLPHOSPHATIDYLINOSITOL_GPI_ANCHOR_BIOSYNTHESIS

KEGG_CARDIAC_MUSCLE_CONTRACTION

KEGG_GLYCINE_SERINE_AND_THREONINE_METABOLISM

KEGG_OOCYTE_MEIOSIS

KEGG_MATURITY_ONSET_DIABETES_OF_THE_YOUNG

KEGG_GLYCOSPHINGOLIPID_BIOSYNTHESIS_LACTO_AND_NEOLACTO_SERIES

KEGG_PRIMARY_IMMUNODEFICIENCY

KEGG_PATHOGENIC_ESCHERICHIA_COLI_INFECTION

KEGG_ASTHMA

KEGG_O_GLYCAN_BIOSYNTHESIS

KEGG_GLYCOSAMINOGLYCAN_BIOSYNTHESIS_KERATAN_SULFATE

KEGG_HYPERTROPHIC_CARDIOMYOPATHY_HCM

KEGG_VASOPRESSIN_REGULATED_WATER_REABSORPTION

KEGG_VIBRIO_CHOLERAE_INFECTION

KEGG_METABOLISM_OF_XENOBIOTICS_BY_CYTOCHROME_P450

KEGG_ABC_TRANSPORTERS

KEGG_INOSITOL_PHOSPHATE_METABOLISM

(e)

FIGURE 2: Identification of the candidate MTGs in the GEO dataset. (a) Venn diagram showing the intersection between DEGs and the survival-related genes. (b, c) LASSO and Cox regression analyses. (d) Boxplot to display the differential expression of genes in MTG signature. (e) The GSEA for the GEO cohort. 


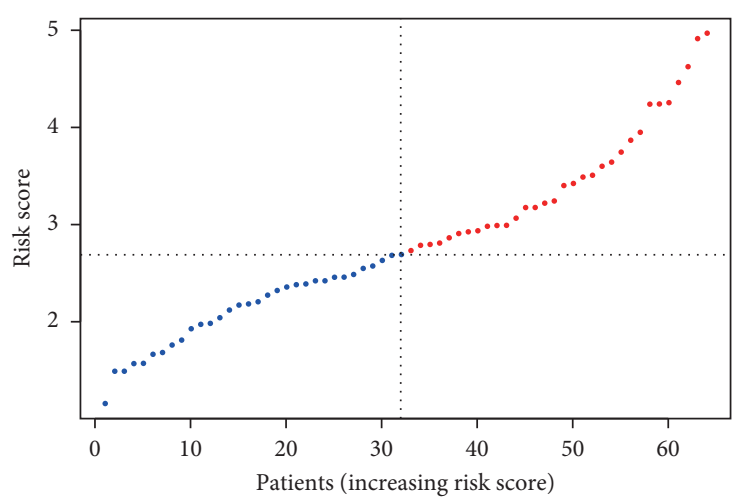

- High risk

- low Risk

(a)

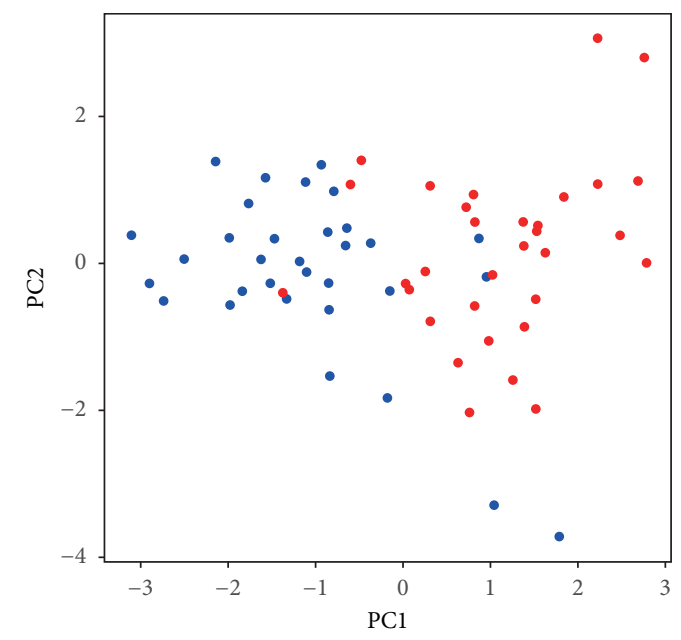

Risk

- high

- low

(c)

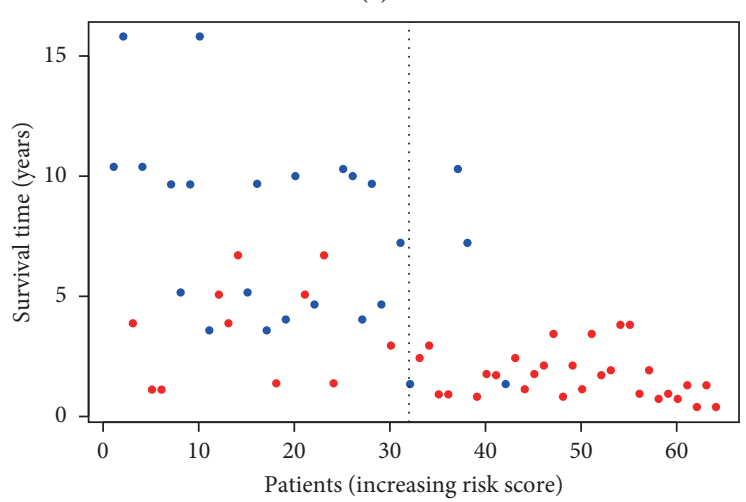

- Dead

- Alive

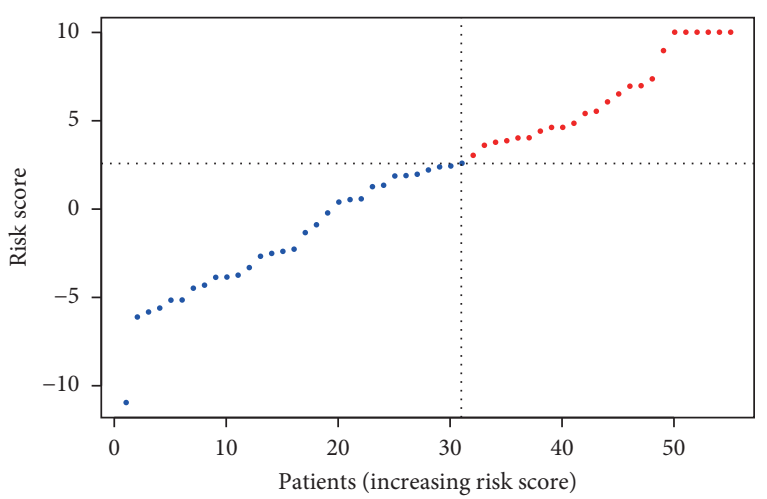

- High risk

- low Risk

(b)

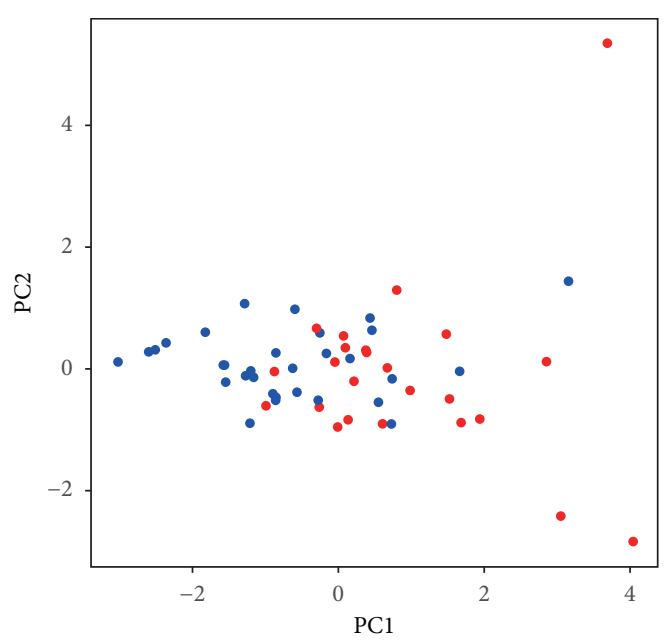

Risk

- high

- low

(d)

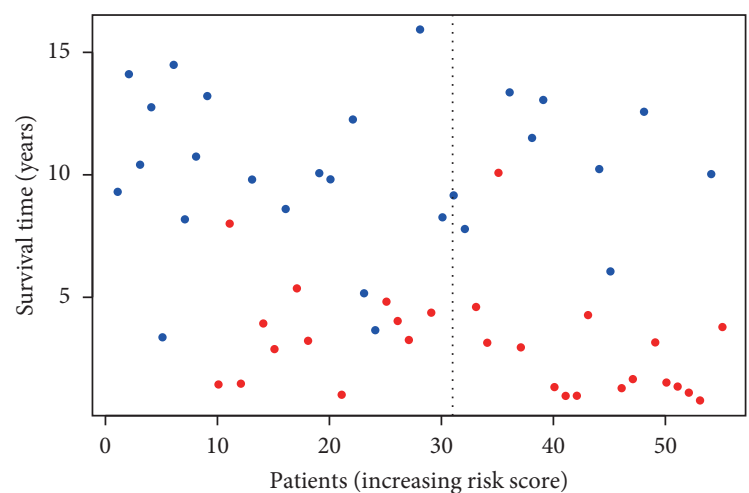

- Dead

- Alive

(e)

Figure 3: Continued. 


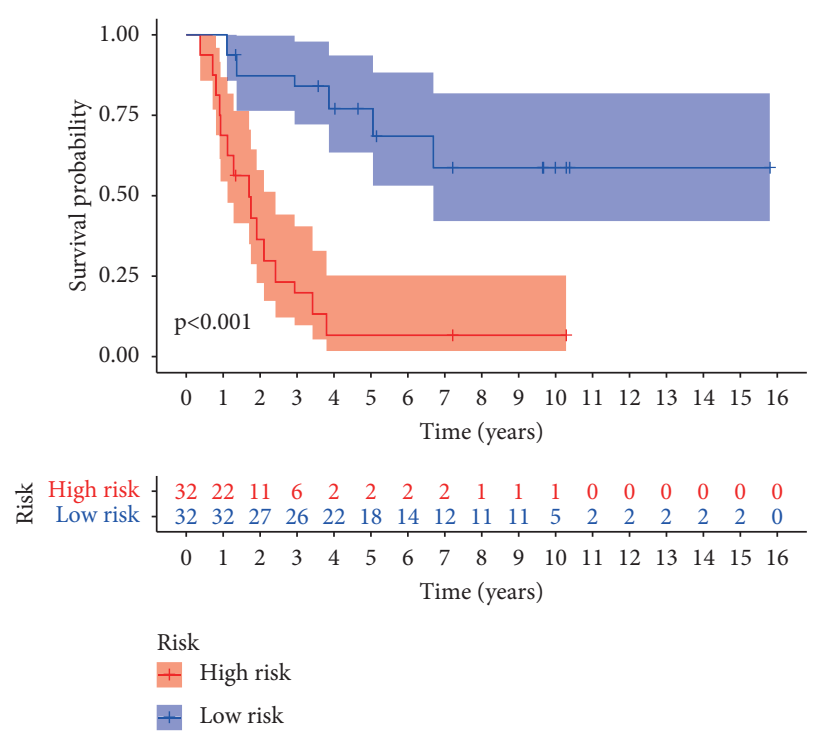

(g)

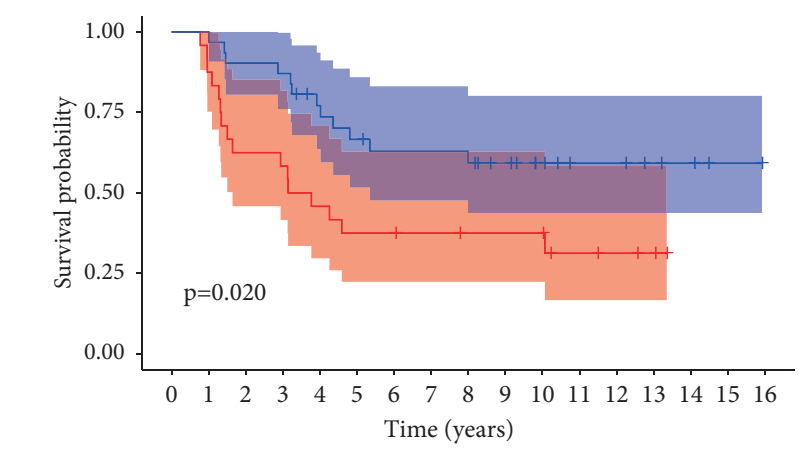

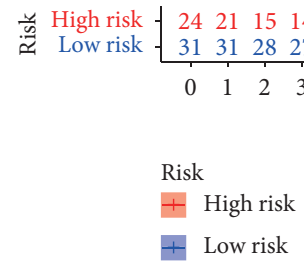

FIgUre 3: Characteristics of the risk score of the MTG signature. The distribution of the risk scores, survival status, PCA analysis, and K-M survival analysis in GEO (a, c, e, g) and ICGC (b, d, f, h) cohorts.

showed that the high-risk group was significantly related to poorer OS $(P<0.05)$.

We also applied the nomogram to explore the 3- and 5year OS of ES patients based on the risk score and other clinical variables (Figure 6(a)). The calibration curve demonstrated satisfactory performance for 3 and 5 years in ES patients (Figures 6(b)-6(c)).

\subsection{Evaluation of Immune Cell Infiltration and Immune} Checkpoints. The relationship between our MTGs and immune infiltration was assessed with the CIBERSORT algorithm. The infiltration profiling and the heatmap of the two groups of 22 immune cells are displayed in Figures 7(a) and 7(b). The correlation of the 22 immune cells is illustrated in the heatmap (Figure $7(\mathrm{c})$ ).

Neutrophils (Figures 8(a) and 8(c)) were highly expressed in the high-risk group $(P<0.001)$. Plasma cells (Figure 8(b)) were highly expressed in the low-risk group $(P=0.042)$. According to the Kaplan-Meier curves (Figure 8(d)), ES patients with higher neutrophil levels exhibited a poorer survival rate.

As for the relationships between immune checkpoint genes (Figure 8(e)) and MTG signature, we found CD40, LGALS9, TMIGD2, ICOSLG, LAIR1, CD48, TNFRSF15, KIR3DL1, and BTNL2 were all significantly highly expressed in the high-risk group while TNFRSF4 was lowly expressed.

\section{Discussion}

Ewing sarcoma is one of the most aggressive sarcomas. Merely $30 \%$ of ES patients with metastasis survive [13]. The lung is one of the most common sites suffering from Ewing sarcoma metastasis while the previous study also showed that colonic Ewing sarcoma could cause liver metastasis [14].
Early diagnosis and treatment can remarkably improve the clinical prognosis of ES, which justifies the need to seek effective biomarkers for the early diagnosis and treatment of ES. Compelling evidence is in support of the finding that MTGs play crucial roles in the development and progression of ES. MTGs have immense potential as promising therapeutic targets and prognostic predictors. However, studies on the prognostic value exploration of MTGs are immature.

Moreover, the outcomes of the survival status and K-M curve demonstrated that the risk score was significantly related to a poorer survival rate in GEO and ICGC datasets. Based on univariate and multivariate Cox analyses, we found that the risk score was of great value as an independent prognostic predictor. ROC curves revealed that our signature could accurately predict the prognosis of ES patients in the two cohorts. Validation procedures showed that the efficiency of the signature was satisfactory in patients with different clinical features. The constructed nomogram could predict the 1-, 3-, and 5-year survival probabilities, which might be useful for personalized treatment. Taken together, all the outcomes indicated that the MTG signature was of good robustness for predicting the prognosis of ES patients. Ren et al. [2] had previously identified immune cell infiltration had a close correlation with ES. Here, we adopted CIBERSORT to explore the roles of the infiltrating immune cells in our signature. The results showed that neutrophils and plasma cells were differentially expressed in the highand low-risk groups. Of note, neutrophil cells were significantly related to poorer OS, which was not the case for plasma cells.

The six genes were identified as follows: PC, CHPT1, and PYGB were oncogenes, while DGKA, CPT1A, and NUDT12 were protective genes. Studies on the relationships between the six genes and Ewing sarcoma are immature. In several cancer tissues, including mammary, lung, gallbladder, and 


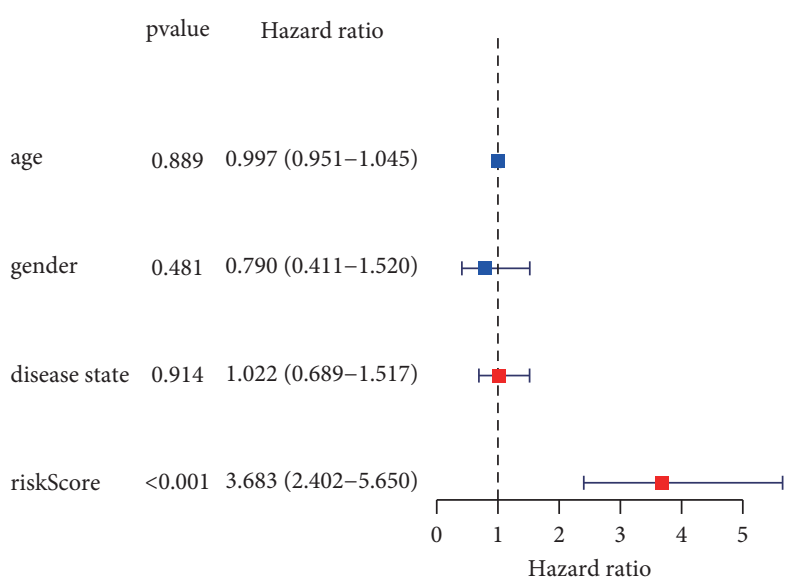

(a)

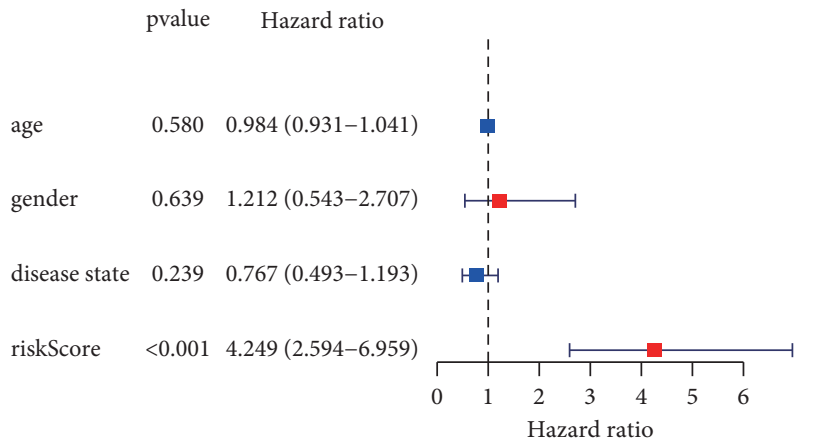

(c)

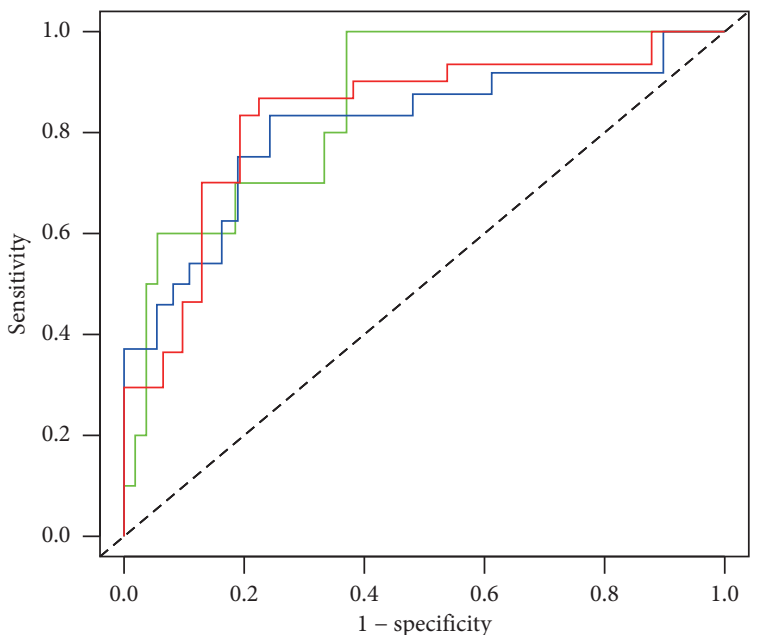

_ AUC at 1 years: 0.856
_ AUC at 2 years: 0.810
_ AUC at 3 years: 0.834

(e)

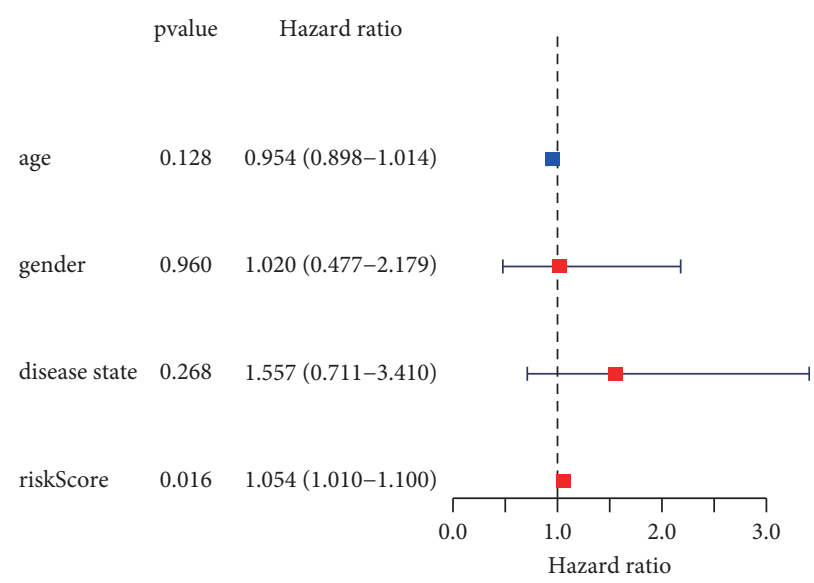

(b)

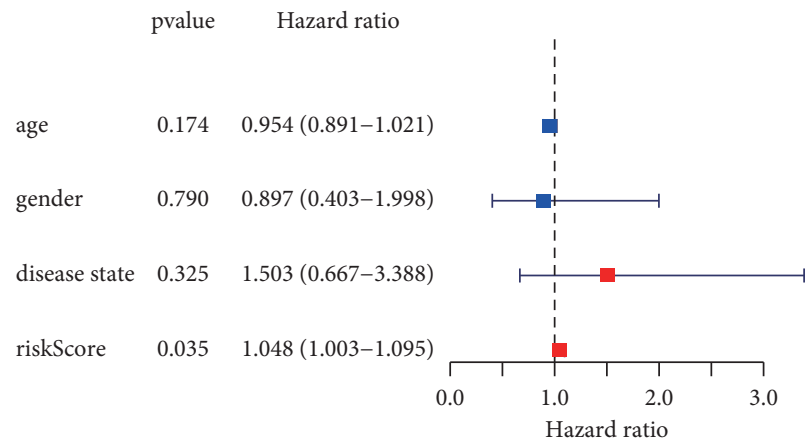

(d)

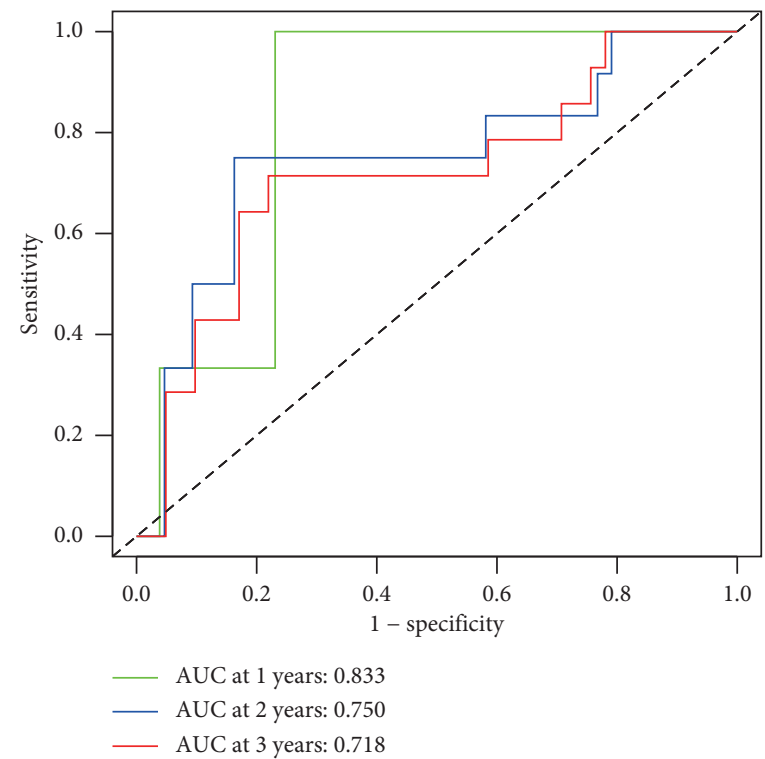

(f)

Figure 4: Continued. 


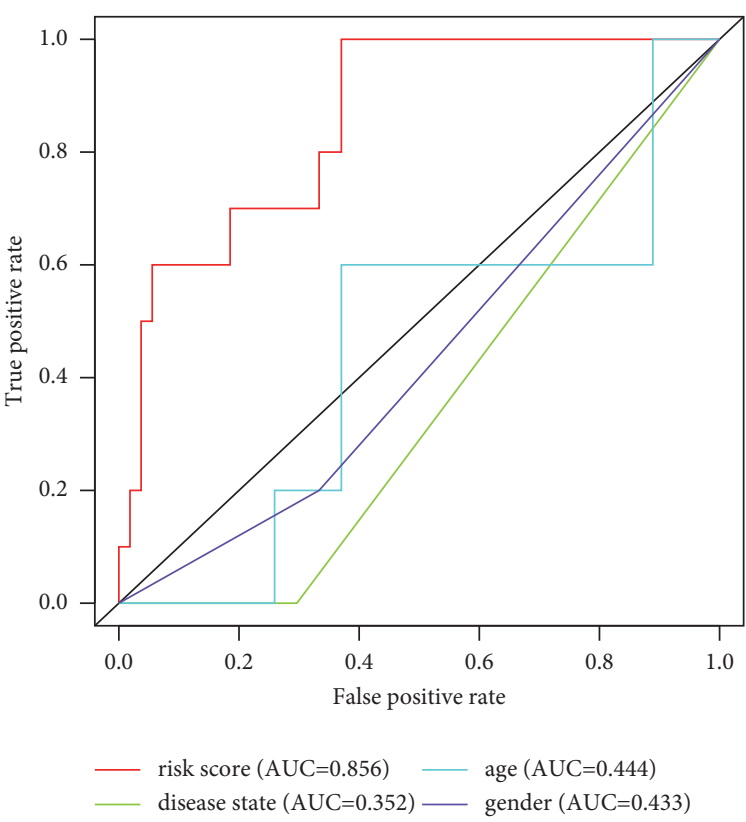

(g)

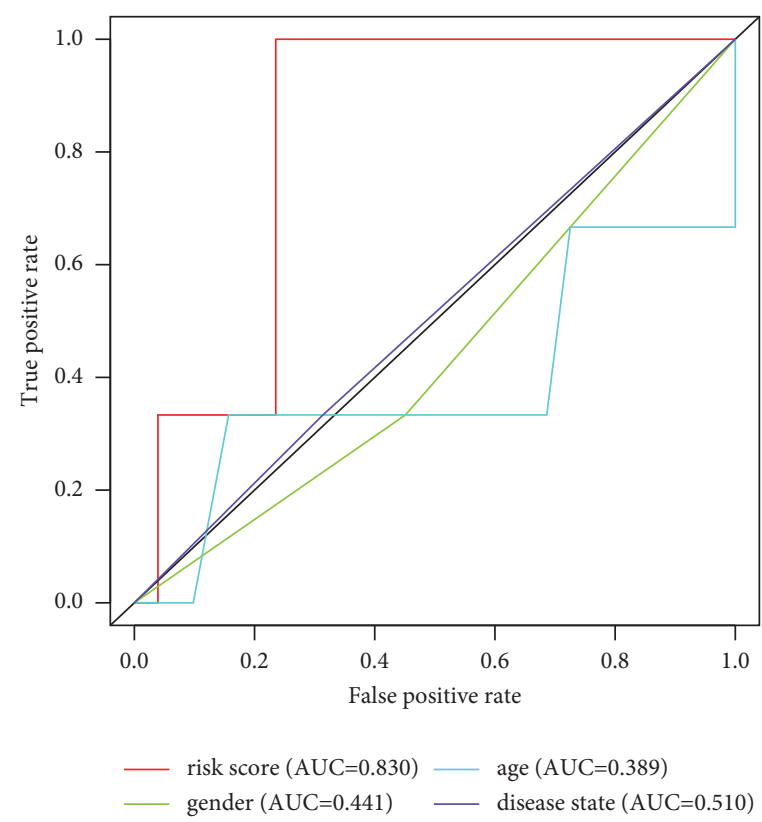

(h)

FIgURE 4: Comprehensive survival analysis of the MTG signature. Univariate and multivariate Cox regression analyses, time-dependent ROC analysis of 1,3 , and 5 years, and time-dependent ROC analysis of risk score and clinical features in GEO (a, c, e, g) and ICGC (b, d, f, h) cohorts.
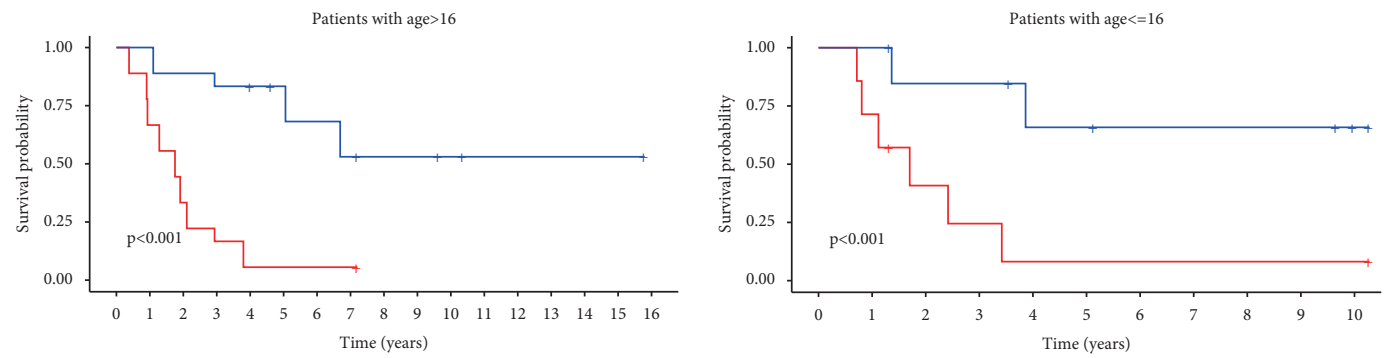

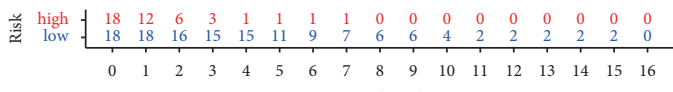

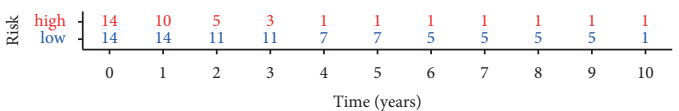

$$
\stackrel{\text { Risk }}{+} \text { high }
$$$$
\text { Time (years) }
$$

$$
\begin{array}{ll}
\text { Risk } \\
+ \text { high } \\
+ \text { low }
\end{array}
$$

(a)

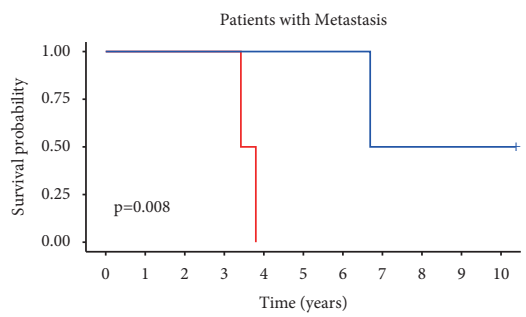

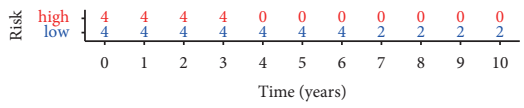

Risk

$\longrightarrow$ high (b)

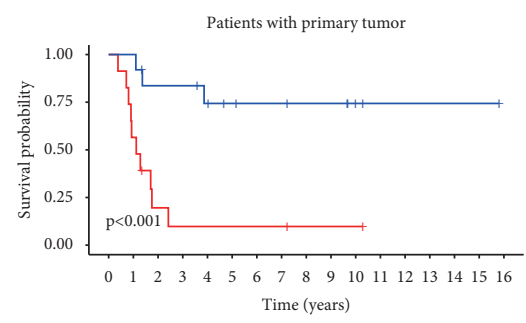

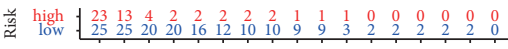

Time (years)

+ high

+ low

(c)

(d)

Figure 5: Continued. 

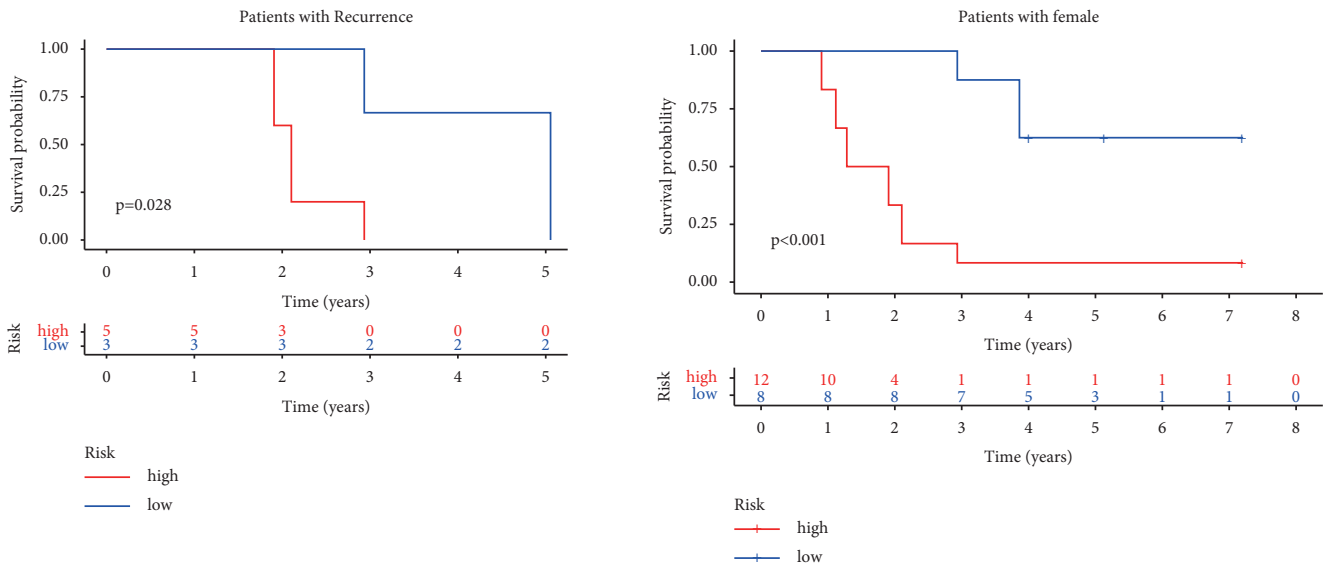

(e)

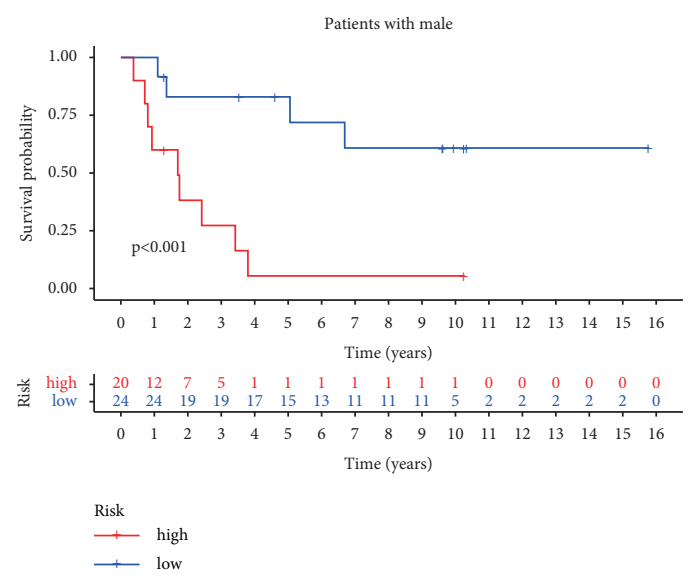

(g)

Figure 5: The efficiency of the MTG signature for prognosis of different subgroups in the GEO cohort. K-M survival analysis for the lowand high-risk groups categorized by clinical variables, comprising age (a, b), metastasis (c), primary tumor (d), recurrence (e), and gender (f, g).

thyroid, Kiesel et al. found that PC was overexpressed as compared with the normal tissue [15]. Other pieces of evidence indicate that PC exerts crucial effects in metastasis, particularly because it connects many metabolism pathways, whereas metastatic tumor cells show an increased need for redox defense and ATP. Mounting evidence shows that CHPT1 is a curative target for prostate cancer [16] and is related to stemness and trastuzumab resistance in breast cancer [17]. Elsewhere, PYGB was also reported to be upregulated in numerous tumors, including gastric cancer, lung cancer, ovarian cancer, and renal cell cancer [18]. Studies have also revealed a close correlation of DGKA, CPT1A, and NUDT12 with numerous tumors [19-21].

Appling a nomogram to cancer prognosis can allow for the interpretation of prediction models and the establishment of numerical possibilities for individualized treatment [22]. We integrated the risk score with other clinicopathological features and established a novel nomogram to assist clinical decision-making. Fang and Chen [23] recently established a nomogram based on the autophagy-related genes, which embodied a favorable effect in hepatocellular carcinoma. In another study, a nomogram containing clinicopathological features and the MTG signature exhibited good results for LUAD prognosis predicting [1]. These data are in support of our nomogram which demonstrated good predictivity potential for 1-, 3-, and 5-year survival of ES patients. In addition, increased studies had revealed the relationship between tumor metabolism and tumor immune $[24,25]$. As one of the immune infiltration cells, neutrophils served as prognosis-related cells and were found to be overexpressed in the high-risk group. According to the immune checkpoint genes, the high-risk group was mainly positively related to the expression levels of immune checkpoint genes.

Although our study presents valid clinical significance, a few limitations cannot be ignored. To begin with, the GEO and ICGC cohorts were derived from the public database, and the clinicopathological features in the two cohorts were incomplete and limited. As such, we needed our dataset to show the effectiveness of the MTG signature. Besides, we did not identify the detailed molecular mechanisms of each MTGs in ES, and further studies are warranted to analyze the details. Lastly, the detailed relationships between the risk score and immune infiltration should be addressed in future. 


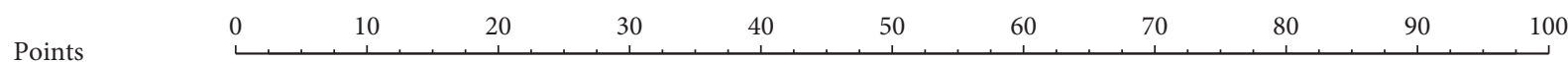

age

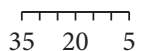

gender $\underset{\substack{\text { Female } \\ \text { Male }}}{\stackrel{2}{c}}$

riskScore

\begin{tabular}{llllllll}
\hline 1 & 1 & 1 & & & & & \\
\hline
\end{tabular}

Total Points

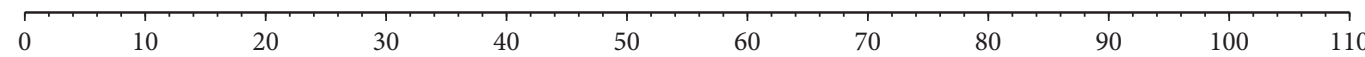

1-Year Survival

$\begin{array}{llllllll}0.9 & 0.8 & 0.7 & 0.6 & 0.5 & 0.4 & 0.3 & 0.2\end{array}$

3-Year Survival

\begin{tabular}{lllllllll}
\hline 0.9 & 0.8 & 0.7 & 0.6 & 0.5 & 0.4 & 0.3 & 0.2 & 0.1
\end{tabular}

5-Year survival

$\begin{array}{lllllllll}0.9 & 0.8 & 0.7 & 0.6 & 0.5 & 0.4 & 0.3 & 0.2 & 0.1\end{array}$

(a)

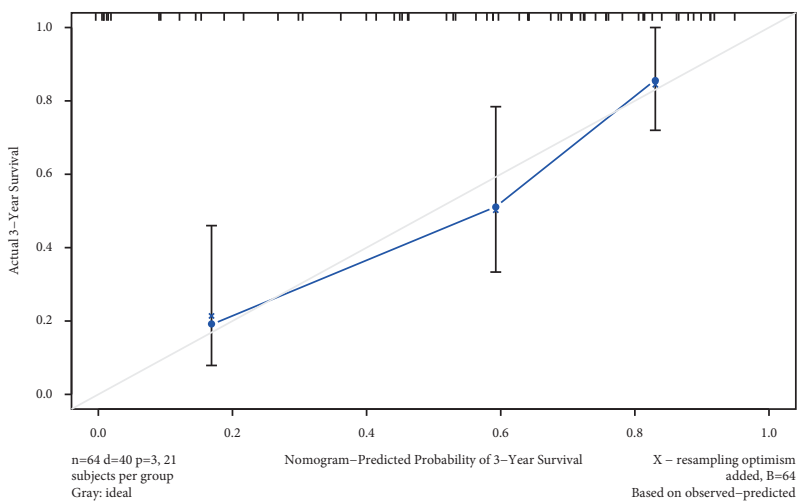

(b)

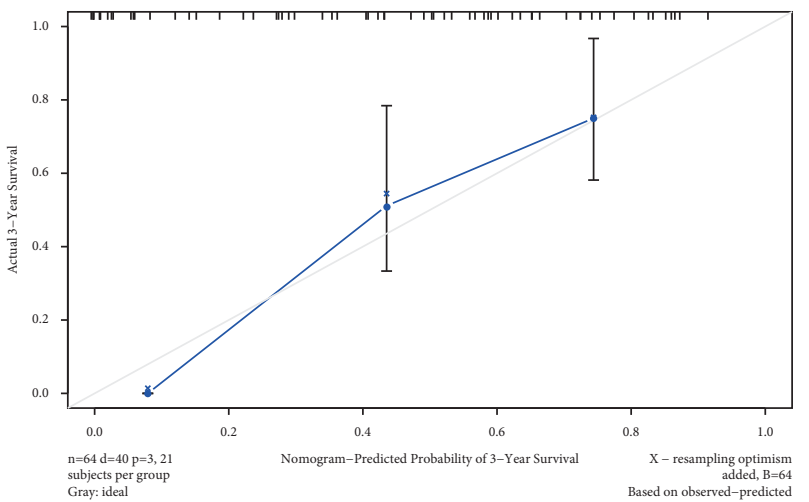

(c)

FIgURE 6: The nomogram for predicting the survival of ES patients in the GEO cohort (a) and 3-year (b) and 5-year (c) survival predicted by calibration plots. 


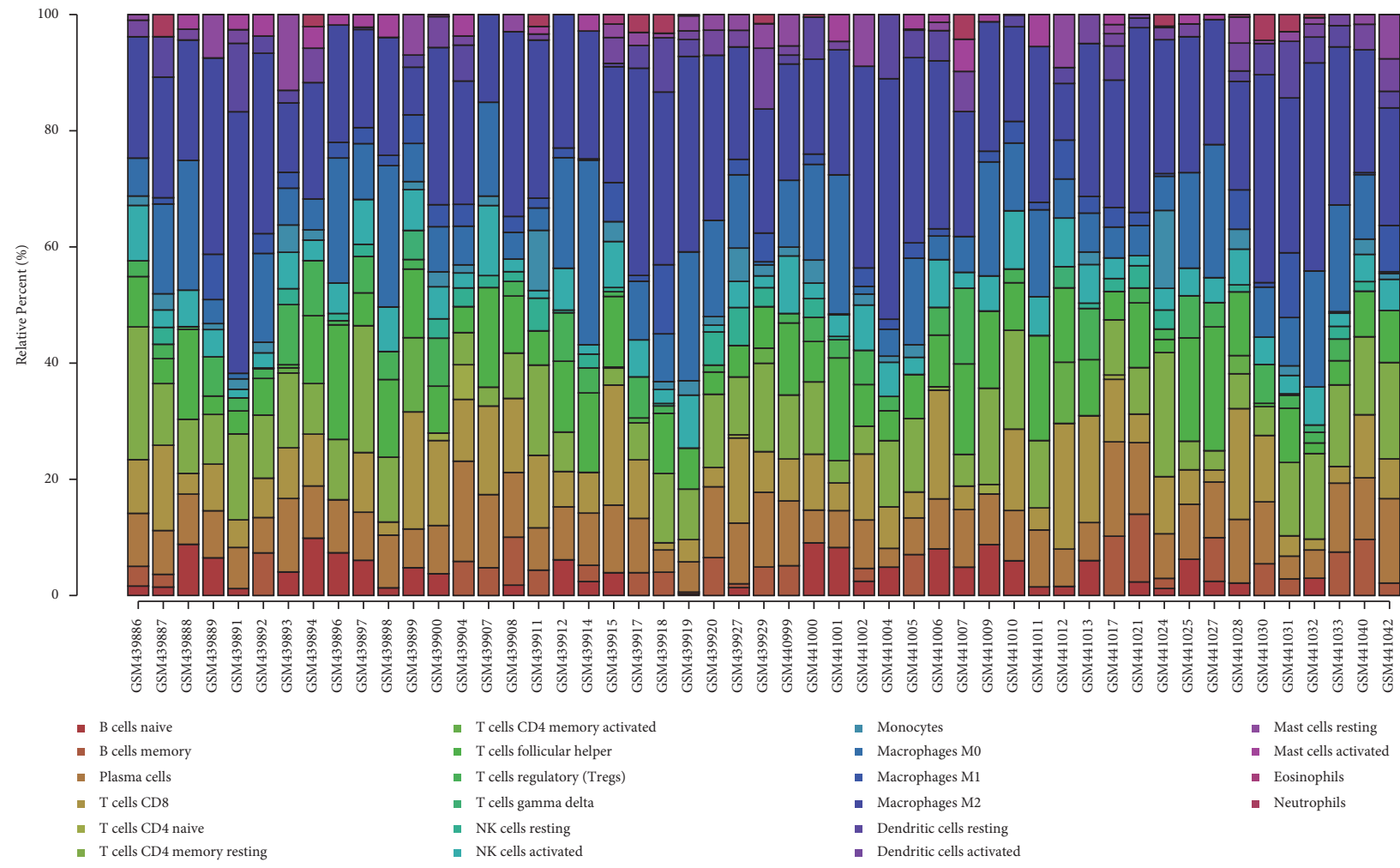

(a)

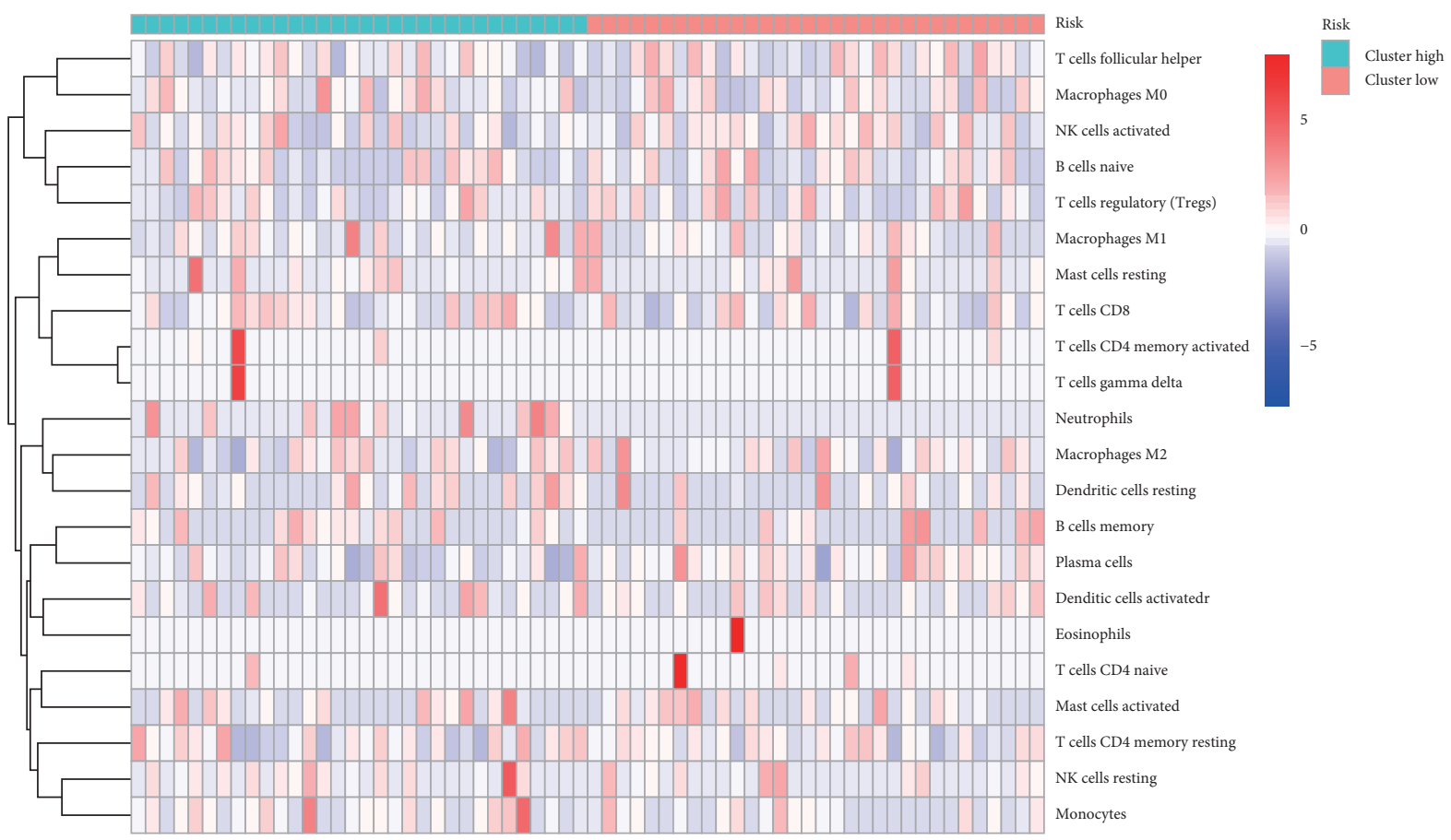

(b)

Figure 7: Continued. 


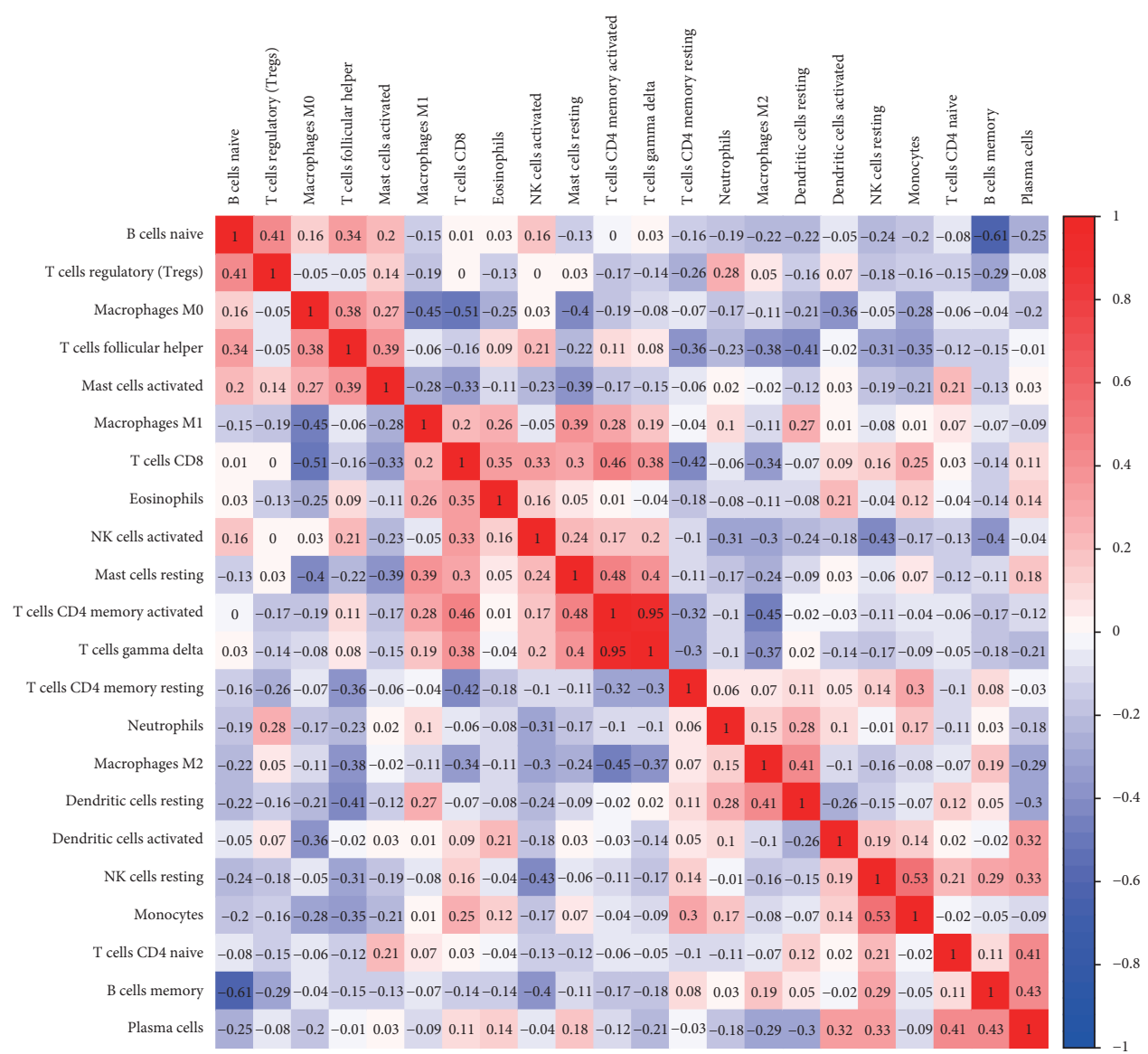

(c)

Figure 7: Twenty-two immune cells proportion (a), heatmap (b), and correlation (c) analysis in the GEO cohort.

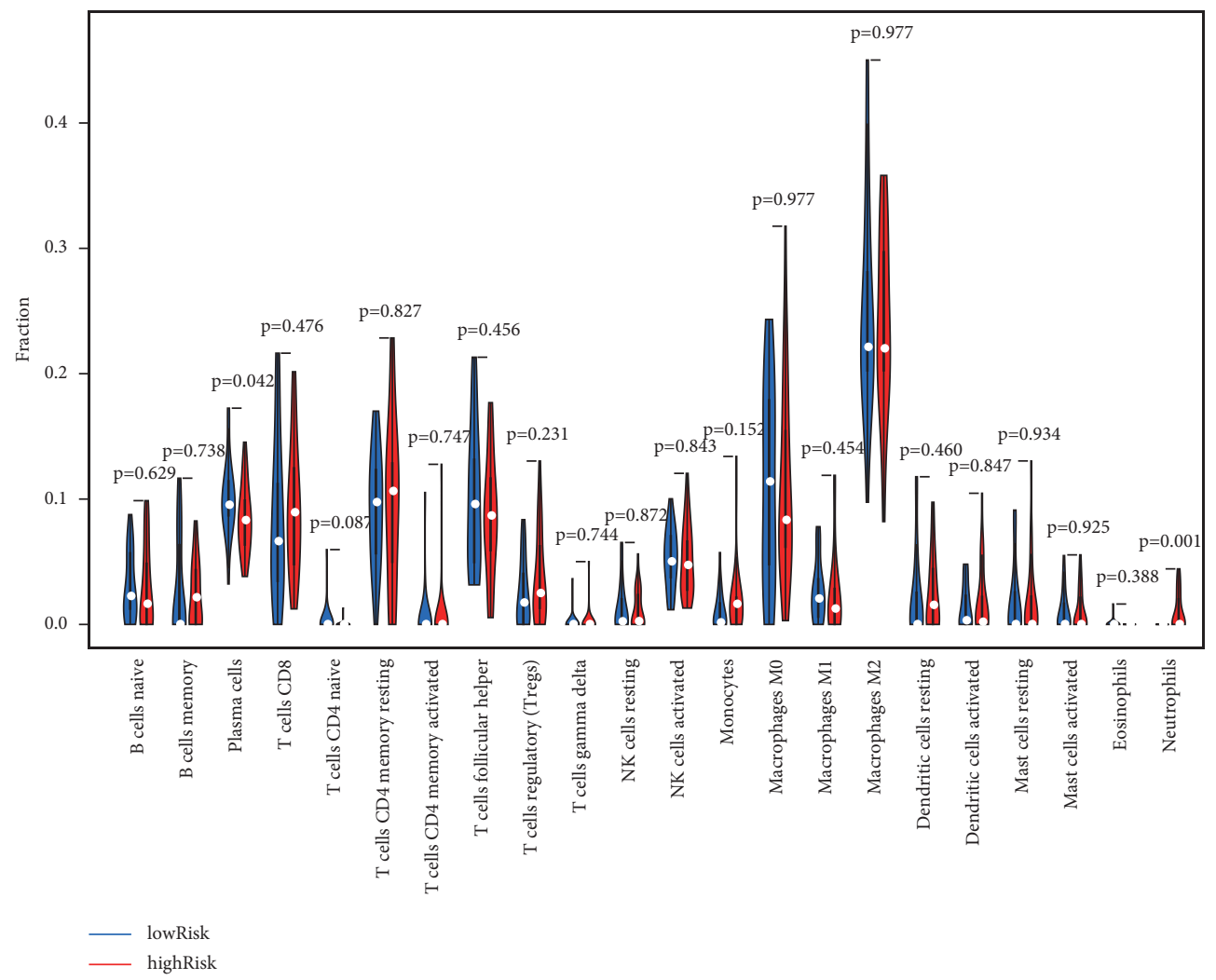

(a)

Figure 8: Continued. 

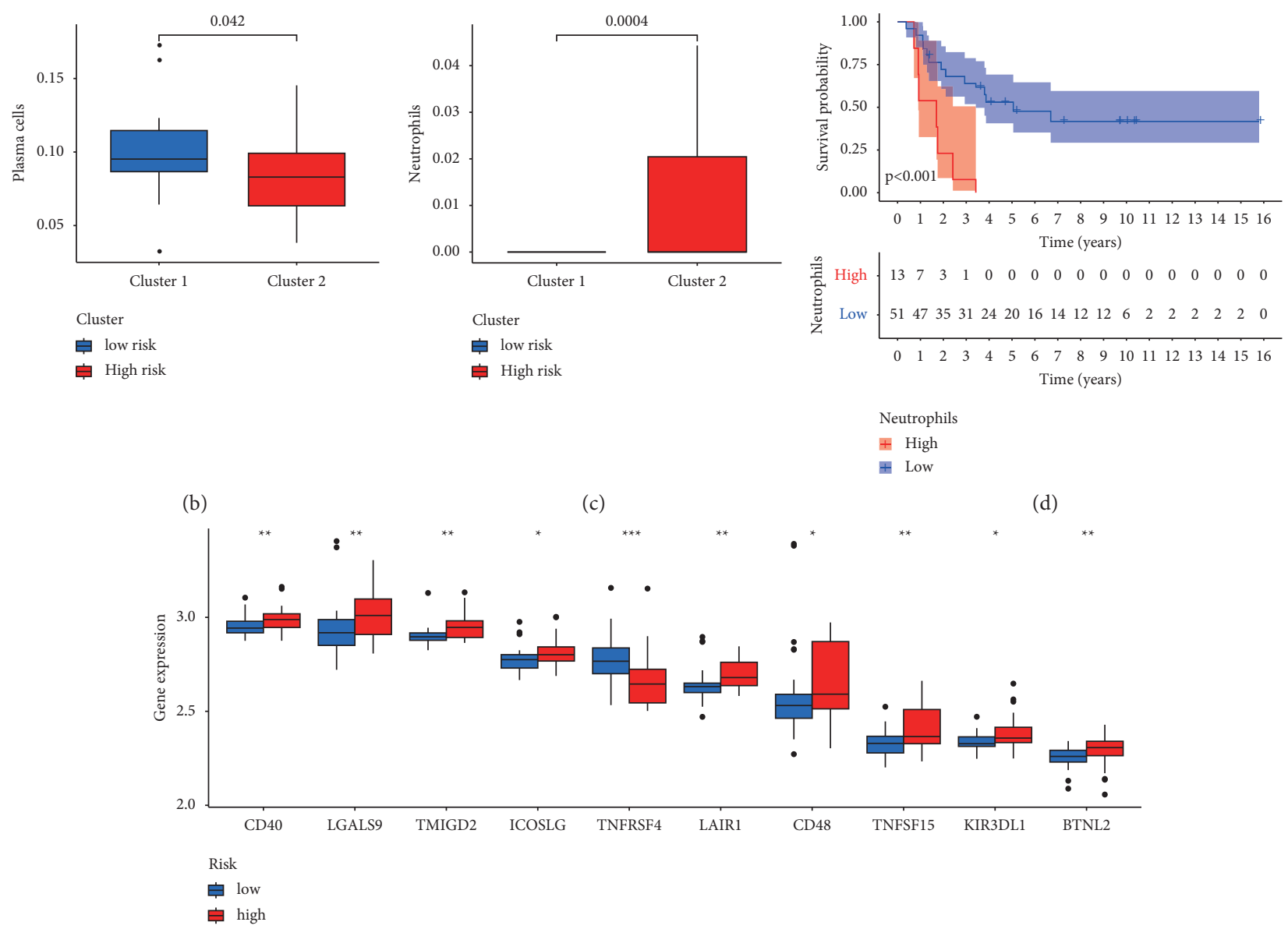

(e)

Figure 8: Evaluation of immune cell infiltration and immune checkpoints. (a) Violin plot showing the composition of immune cells between the low- and high-risk groups (low: blue vs. high: red). Box plot showing the differential immune cells: neutrophils (b) and plasma cells (c). (d) K-M survival analysis of the neutrophils in the low- and high-risk groups. (e) Box plot showing differential expression of immune checkpoints genes.

\section{Conclusion}

In conclusion, the MTG signature developed in this work displayed an upstanding performance as an independent factor for predicting the prognosis of ES patients. The signature has been validated in an independent cohort. Also, the MTG signature-related nomogram can predict 3- and 5year survival outcomes accurately. Overall, this study presents six genes with potential roles as prognostic indicators and therapeutic targets for ES.

\section{Abbreviations}

ES: $\quad$ Ewing sarcoma

LDH: Lactate dehydrogenase

DEGs: Differentially expressed genes

MTG: Metabolism-related gene

ICGC: International Cancer Genome Consortium

ssGSEA: Single-sample gene set enrichment analysis

LASSO: Least absolute shrinkage and selection operator

GSEA: Gene Set Enrichment Analysis

OS: Overall survival
PCA: Principal component analysis.

\section{Data Availability}

The datasets analyzed in this study can be derived from https://www.ncbi.nlm.nih.gov/geo/query/acc.cgi?acc=GSE1 7679 and https://dcc.icgc.org/repositories. The codes in this study are available from the corresponding author on reasonable request.

\section{Conflicts of Interest}

The authors declare no conflicts of interest.

\section{Authors' Contributions}

ZYC and HL designed the study; ZYC wrote the manuscript. HY and JB obtained the two cohorts. ZY performed statistical analyses. HL revised the manuscript. HY and QC generated all figures and prepared the supplementary information. 


\section{Acknowledgments}

The authors sincerely appreciate all scientists who contributed to the data in the TCGA and GEO database and MSigDB database. This study was funded by the Natural Science Foundation of Hunan Province (no. 2020JJ5331), China.

\section{Supplementary Materials}

944 metabolism-associated genes are shown. (Supplementary Materials)

\section{References}

[1] L. He, J. Chen, F. Xu, J. Li, and J. Li, "Prognostic implication of a metabolism-associated gene signature in lung adenocarcinoma," Molecular Therapy - Oncolytics, vol. 19, pp. 265-277, 2020.

[2] E.-h. Ren, Y.-j. Deng, W.-h. Yuan, Z.-l. Wu, G.-z. Zhang, and Q.-q. Xie, "An immune-related gene signature for determining Ewing sarcoma prognosis based on machine learning," Journal of Cancer Research and Clinical Oncology, vol. 147, no. 1, pp. 153-165, 2021.

[3] Y. Yang, Y. Ma, H. Gao et al., "A novel HDGF-ALCAM axis promotes the metastasis of Ewing sarcoma via regulating the GTPases signaling pathway," Oncogene, vol. 40, no. 4, pp. 731-745, 2021.

[4] E. Koustas, P. Sarantis, M. V. Karamouzis, P. Vielh, and S. Theocharis, "The controversial role of autophagy in ewing sarcoma pathogenesis-current treatment options," Biomolecules, vol. 11, no. 3, p. 355, 2021.

[5] M. G. Vander Heiden, L. C. Cantley, and C. B. Thompson, "Understanding the Warburg effect: the metabolic requirements of cell proliferation," Science, vol. 324, no. 5930, pp. 1029-1033, 2009.

[6] Z. He, C. Wang, H. Xue, R. Zhao, and G. Li, "Identification of a metabolism-related risk signature associated with clinical prognosis in glioblastoma using integrated bioinformatic analysis," Frontiers in oncology, vol. 10, p. 1631, 2020.

[7] J. Chen, Y. Yu, H. Li et al., "Long non-coding RNA PVT1 promotes tumor progression by regulating the miR-143/HK2 axis in gallbladder cancer," Molecular Cancer, vol. 18, no. 1, p. 33, 2019.

[8] M. G. Vander Heiden and R. J. DeBerardinis, "Understanding the intersections between metabolism and cancer biology," Cell, vol. 168, no. 4, pp. 657-669, 2017.

[9] J. M. Tanner, C. Bensard, P. Wei et al., "EWS/FLI is a master regulator of metabolic reprogramming in ewing sarcoma," Molecular Cancer Research, vol. 15, no. 11, pp. 1517-1530, 2017.

[10] C. Yeung, A. E. Gibson, S. H. Issaq et al., "Targeting glycolysis through inhibition of lactate dehydrogenase impairs tumor growth in preclinical models of ewing sarcoma," Cancer Research, vol. 79, no. 19, pp. 5060-5073, 2019.

[11] G. Chao-Yang, T. Rong, S. Yong-Qiang et al., "Prognostic signatures of metabolic genes and metabolism-related long non-coding RNAs accurately predict overall survival for osteosarcoma patients," Frontiers in cell and developmental biology, vol. 9, Article ID 644220, 2021.

[12] A. M. Newman, C. L. Liu, M. R. Green et al., "Robust enumeration of cell subsets from tissue expression profiles," Nature Methods, vol. 12, no. 5, pp. 453-457, 2015.
[13] T. G. P. Grünewald, F. Cidre-Aranaz, D. Surdez et al., "Ewing sarcoma," Nature Reviews Disease Primers, vol. 4, no. 1, p. 5, 2018.

[14] P. Parcesepe, G. Giordano, C. Zanella et al., "Colonic Ewing Sarcoma/PNET associated with liver metastases: a systematic review and case report," Pathology, Research \& Practice, vol. 215, no. 2, pp. 387-391, 2019.

[15] V. A. Kiesel, M. P. Sheeley, M. F. Coleman et al., "Pyruvate carboxylase and cancer progression," Cancer \& Metabolism, vol. 9 , no. 1, p. 20, 2021.

[16] I. R. Schlaepfer, L. Rider, L. U. Rodrigues et al., "Lipid catabolism via CPT1 as a therapeutic target for prostate cancer," Molecular Cancer Therapeutics, vol. 13, no. 10, pp. 2361-2371, 2014.

[17] J. Han, H. Qu, M. Han et al., "MSC-induced lncRNA AGAP2AS1 promotes stemness and trastuzumab resistance through regulating CPT1 expression and fatty acid oxidation in breast cancer," Oncogene, vol. 40, no. 4, pp. 833-847, 2021.

[18] Y. Zhou, Z. Jin, and C. Wang, "Glycogen phosphorylase B promotes ovarian cancer progression via $\mathrm{Wnt} / \beta$-catenin signaling and is regulated by miR-133a-3p," Biomedicine \& Pharmacotherapy, vol. 120, Article ID 109449, 2019.

[19] C. L. Dominguez, D. H. Floyd, A. Xiao et al., "Diacylglycerol kinase $\alpha$ is a critical signaling node and novel therapeutic target in glioblastoma and other cancers," Cancer Discovery, vol. 3, no. 7, pp. 782-797, 2013

[20] Z. Tan, L. Xiao, M. Tang et al., "Targeting CPT1A-mediated fatty acid oxidation sensitizes nasopharyngeal carcinoma to radiation therapy," Theranostics, vol. 8, no. 9, pp. 2329-2347, 2018.

[21] Y. Zhou, X. Cheng, F. Zhang et al., "Integrated multi-omics data analyses for exploring the co-occurring and mutually exclusive gene alteration events in colorectal cancer," Human Mutation, vol. 41, no. 9, pp. 1588-1599, 2020.

[22] A. Iasonos, D. Schrag, G. V. Raj, and K. S. Panageas, "How to build and interpret a nomogram for cancer prognosis," Journal of Clinical Oncology, vol. 26, no. 8, pp. 1364-1370, 2008.

[23] Q. Fang and H. Chen, "Development of a novel autophagyrelated prognostic signature and nomogram for hepatocellular carcinoma," Frontiers in oncology, vol. 10, Article ID 591356, 2020.

[24] X.-N. Wu, D. Su, Y.-D. Mei et al., "Identified lung adenocarcinoma metabolic phenotypes and their association with tumor immune microenvironment," Cancer Immunology, Immunotherapy, vol. 70, no. 10, pp. 2835-2850, 2021.

[25] S. Yu, C. Hu, L. Cai et al., "Seven-gene signature based on glycolysis is closely related to the prognosis and tumor immune infiltration of patients with gastric cancer," Frontiers in oncology, vol. 10, p. 1778, 2020. 